\title{
Frontières
}

\section{Douleur et anthropologie}

Esquisses

\section{David Le Breton}

Volume 17, numéro 2, printemps 2005

Surtout, ne pas souffrir

URI : https://id.erudit.org/iderudit/1073483ar

DOI : https://doi.org/10.7202/1073483ar

Aller au sommaire du numéro

\section{Éditeur(s)}

Université du Québec à Montréal

ISSN

1180-3479 (imprimé)

1916-0976 (numérique)

Découvrir la revue

Citer cet article

Le Breton, D. (2005). Douleur et anthropologie : esquisses. Frontières, 17(2),

7-12. https://doi.org/10.7202/1073483ar
Résumé de l'article

Il n'y a pas de douleurs sans souffrance. La douleur brise l'existence à proportion de la souffrance qu'elle induit chez le sujet. Différents facteurs influent sur ce ressenti : l'appartenance sociale, culturelle, les circonstances qui la produisent, le récit qu'en fait le sujet, etc. Ce texte suggère un certain nombre de champs de recherche dont il esquisse les données. d'utilisation que vous pouvez consulter en ligne.

https://apropos.erudit.org/fr/usagers/politique-dutilisation/ 
Résumé

Il n'y a pas de douleurs sans souffrance. La douleur brise l'existence à proportion de la souffrance qu'elle induit chez le sujet. Différents facteurs influent sur ce ressenti: I'appartenance sociale, culturelle, les circonstances qui la produisent, le récit qu'en fait le sujet, etc. Ce texte suggère un certain nombre de champs de recherche dont il esquisse les données.

Mots clés: souffrance - douleur anthropologie.

\section{Abstract}

There is no pain without suffering. Pain destroies in the way suffering hurts the individual. Different matters influence the feeling of pain (suffering): social and cultural affiliations, circumstances that surround the narrative done by the individual, and so on. This text suggests a lot of fields of investigations.

Keywords: suffering - pain anthropology.

\section{DOULEUR ET ANTHROPOLOGIE ESQUISSES}

\begin{abstract}
ET NOUS NE CONNAÎTRONS VRAIMENT LA DOULEUR PHYSIQUE QUE QUAND NOUS SAURONS CE QUI FAIT L'INDIVIDUEL DANS UN SYSTĖME MORPHOLOGIQUEMENT LE MÊME POUR TOUT LE MONDE, QUAND NOUS AURONS ANALYSÉ [...] LA DOULEUR VIVANTE. LA DOULEUR PHYSIQUE EST CERTAINEMENT DOMINÉE PAR UN ÉLÉMENT MORAL, PAR L'ÉNERGIE, PAR LA VOLONTÉ. MAIS, COMMENT CES FACTEURS INTERVIENNENT-ILS? NE FONT-ILS QUE FREINER L'EXPRESSION, LA MIMIQUE DU VISAGE? OÜ SONT-ILS CAPABLES DE DIMINUER L'ACUITÉ DE NOS PERCEPTIONS DOULOUREUSES?
\end{abstract}

RENÉ LERICHE, CHIRURGIE DE LA DOULEUR, MASSON, 1937, P. 10.

\begin{abstract}
David Le Breton, Ph.D.,
anthropologue, Université Marc-Bloch de Strasbourg.
\end{abstract}

La douleur, qu'elle soit provoquée par la maladie ou par les séquelles d'un accident, rompt les amarres qui attachaient l'individu à ses activités familières, elle rend difficile la relation avec les proches, élimine ou diminue le goût de vivre. Aucun refuge n'échappe à sa virulence. Elle paralyse l'activité de pensée ou l'exercice de la vie. Elle pèse sur le jeu du désir, le lien social. Déchirure de soi qui brise l'évidence de la relation au monde, elle force au dualisme en contraignant à vivre son corps comme un fardeau, une altérité insupportable. En perdant la confiance élémentaire en son corps, l'individu perd la confiance en soi et dans le monde, son propre corps s'érige en ennemi sournois et implacable menant sa vie propre. La douleur aiguise le sentiment de solitude, elle contraint l'individu à une relation insistante avec sa peine. Enfouie dans l'obscurité de la chair, elle est réservée à la seule délibération intime. Elle suscite le cri, la plainte, le gémissement, les pleurs ou le silence, autant de défaillances de la parole et de la pensée; elle brise la voix et la rend méconnaissable. La douleur immerge dans un univers inaccessible à tout autre. Souffrir comme l'autre ne suffit pas à dissiper l'éloignement et à nouer d'emblée une communauté de destin, car la douleur isole et retient chacun dans ses griffes particulières. L'homme qui souffre tend à s'éloigner des autres. Limpression que nul ne le comprend, qu'une telle souffrance est inaccessible à leur compassion ou à leur simple entendement, accentue encore cette tendance. La douleur est une expérience forcée et violente des limites de la condition humaine, elle inaugure un mode de vie, un emprisonnement en soi qui ne laisse guère de répit. La douleur imprègne l'existence sur le mode de la violation. Un organe, un tissu lésé, une fonction jusqu'alors diluée dans l'évidence du corps, retient l'attention exclusive de l'individu, prend le pas sur les 
choses essentielles de la vie ordinaire au point même d'anéantir, quand elle se fait chronique, presque tout intérêt envers le monde et les autres, devenant une sorte d'occupation à plein temps.

\section{LA PLURALITÉ DE LA DOULEUR}

La plupart du temps le médecin trouve un ancrage physiologique et une parade antalgique. Mais en cas d'échec, et particulièrement de douleur chronique, le médecin ou l'entourage doivent savoir interroger la signification de la plainte. La douleur est alors dans la vie avant de faire corps avec l'individu. Elle est ainsi parfois insaisissable car elle relève de la dimension symbolique et la raison physiologique n'en épuise pas toute la signification. Les médecins connaissent ces douleurs qui se renversent en plaisir dans l'hystérie ou le masochisme, ces douleurs sans cause apparente, ces plaintes sans commune mesure avec la lésion, ces douleurs persistantes après guérison, ou à l'inverse ces lésions graves sans douleur, ces blessures terribles que s'infligent des psychotiques. La dimension ambiguë, mais symbolique, de la douleur nourrit des symptômes ou des plaintes sans que rien n'indique que la chair soit altérée. Il en est ainsi des innombrables malades dits «fonctionnels» qui composent un fort pourcentage des consultations enregistrées par les professionnels de santé. La souffrance est là, elle pèse sur l'existence sans que nulle lésion organique soit repérable avec les outils de diagnostic sophistiqués de la médecine moderne. Le symptôme est un écran, le corps, une voie royale pour faire entendre un manque à être qui ronge le rapport au monde.

Souvent demande de reconnaissance, d'attention, venant rompre un sentiment personnel d'insignifiance ou de solitude (Melsack et Wall, 1989; Balint, 1966), la douleur est l'indice d'une souffrance existentielle qui résonne dans la chair et autorise socialement un contact, un réconfort. La clinique des enfants ou des vieillards est souvent révélatrice à ce propos. La douleur est toujours le fait d'une histoire personnelle. Entre mal de vivre et mal du corps, elle oscille, unissant l'un à l'autre de manière subtile et nécessaire, ou parfois de manière folle et cruelle. Aucune loi physiologique ne la fonde en vérité, elle est multiple: instance paradoxale de sauvegarde, preuve répétée de l'existence, substitut d'amour pour pallier l'absence, moyen de pression sur l'autre, garantie d'une revendication, mode d'expiation, etc. Elle communique une information, non seulement sur l'état physique ou moral de l'individu, mais aussi sur l'état de ses relations avec les autres, et surtout avec les autres intériorisés à la manière d'une histoire enfouie. Dans nombre de cas, la douleur remplit à l'évidence, de façon immédiate ou secondaire, une fonction anthropologique de maintien de l'identité ${ }^{1}$. Tous ces troubles révèlent que la relation de l'homme à son corps est un enchevêtrement complexe de données existentielles et physiologiques. Soustraire l'une ou l'autre de ces influences serait perdre toute prise sur le réel du malade.

D'autres circonstances sont propices à la fabrication de symptômes où la douleur joue un rôle de garant d'authenticité. Lorsque la sexualité est vécue dans la culpabilité ou le déplaisir, à l'adolescence notamment, la douleur (migraines, maux de ventre, etc.) légitime la peur ou le refus du passage à l'acte. Elle peut être également un refuge éloignant la crainte d'un changement perçu plus ou moins consciemment comme une menace pour le sentiment d'identité. Au prix de la peine, de la privation, de l'appréhension, l'individu satisfait partiellement à une défense de soi, évite de s'exposer à une situation qui lui serait plus pénible encore. Cette expérience paraît préférable au renoncement ou à la rupture d'un nœud d'identité.

Sans autre aveu que la parole souffrante, la douleur ne fournit pas de preuves tangibles, son évaluation repose seulement sur les déclarations du patient. Elle est réelle dans la mesure où elle est ressentie et meurtrit la relation au monde. La trace organique du mal est un aspect d'une réalité plus insaisissable: ce n'est pas une lésion qui souffre ou les afférences sympathiques d'un organe mutilé, mais un homme au singulier. La douleur est en ce sens une objection radicale au dualisme entre le corps, lieu de la juridiction spécifique de la médecine, et le reste, l'âme ou le psychisme dont l'intérêt serait négligeable. L'échec relativement courant à identifier les racines de la douleur chez des patients dont l'existence est profondément meurtrie est l'un des effets pervers d'une science médicale toute centrée sur le corps et ses signes. La médecine est un savoir du corps malade et de ses processus, elle est nettement moins un savoir sur l'homme souffrant. Historiquement, elle s'est constituée sur la base du dualisme, écartant l'individu avec méthode pour exercer sa sagacité sur le corps humain dont elle démembrait la structure, et inventoriait les processus pathologiques ${ }^{2}$. L'homme dans sa singularité souffrante n'est pas inclus d'emblée dans ce paradigme. L'impossibilité de faire avouer le corps sur la réalité de la douleur de l'individu est l'une des conséquences du refoulement de l'homme dans l'épistémologie médicale. De même que l'homme est le reste de la maladie dans certaines procédures de prise en charge, la douleur introuvable est le résidu d'une médecine qui établit sa souveraineté sur un corps qu'elle regarde de trop près dans

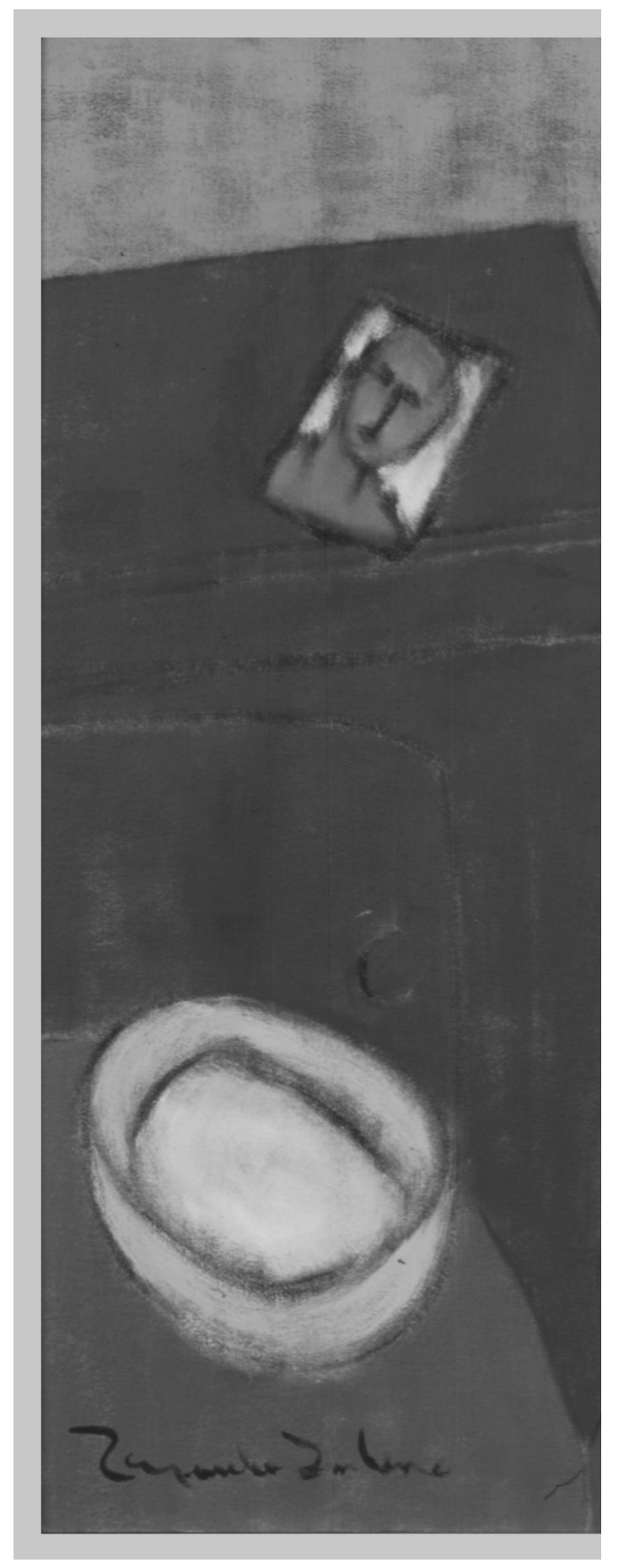

l'oubli du visage singulier du malade. La douleur ne se laisse pas emprisonner dans la chair, elle implique un homme souffrant. Elle rappelle que les modalités physiques de la relation de l'homme au monde prennent corps au sein du lien social, c'est-à-dire au cœur de la dimension symbolique.

\section{DE LA DOULEUR À LA SOUFFRANCE}

Une douleur n'est pas le décalque dans la conscience d'une effraction organique, elle mêle le corps et le sens. Elle est somatisation et sémantisation. Elle n'est pas une sensation mais une perception, c'est-à-dire la confrontation d'un événement corporel à 


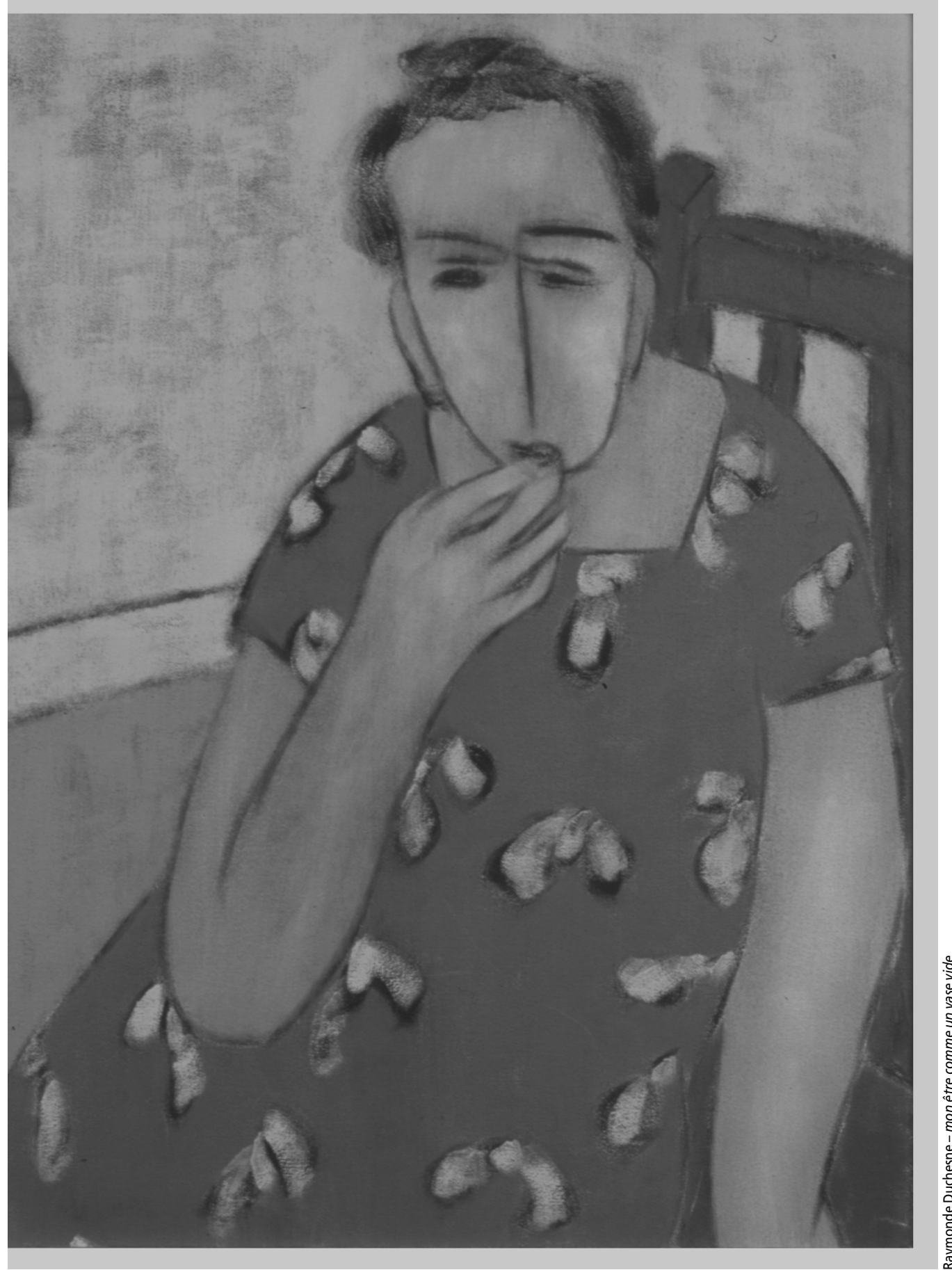

un univers de sens et de valeur. Le ressenti n'est pas l'enregistrement d'une affection, mais la résonance en soi d'une atteinte réelle ou symbolique. Le sens n'est pas contenu dans les choses, il s'instaure dans la relation avec les choses, et dans le débat noué avec les autres pour leur définition, dans la complaisance ou non du monde à se ranger dans ces catégories. Sentir le monde, même la douleur, est une autre manière de le penser, de le transformer de sensible en intelligible. L'expérience humaine tient d'abord aux significations avec lesquelles le monde est vécu, car ce dernier ne se donne pas sous d'autres auspices. Dès lors

\section{mon être}

\section{comme un vase vide}

qu'il a cessé d'être lui-même. Toute douleur induit une métamorphose, elle transforme en profondeur pour le meilleur ou pour le pire l'homme qui est frappé par elle (Le Breton, 1995).

La douleur entraîne un retentissement dans la relation de l'homme au monde. Elle n'est pas cantonnée à un organe ou à une fonction, elle est aussi morale. Le mal de dent n'est pas dans la dent, il est dans la vie, il altère toutes les activités de l'homme, même celles qu'il affectionne. L'homme souffre dans toute l'épaisseur de son être, de son histoire. On distingue traditionnellement la douleur, atteinte de la chair, et la souffrance, atteinte de la psyché. Cette distinction commode est simultanément ambiguë en ce qu'elle oppose sans ciller le corps et l'homme comme deux réalités distinctes, faisant ainsi de l'individu le produit d'un collage surréaliste entre une âme et un corps. La douleur n'écrase pas le corps, \& elle écrase l'individu, elle rompt l'évidence de son rapport au monde, elle brise l'écoulement de la vie quotidienne et altère la relation aux autres.

La douleur est toujours contenue dans une souffrance, elle est d'emblée un pâtir, une agression à supporter. L'anthropologie traite donc de la souffrance en tant qu'elle est la résonance intime d'une douleur. La souffrance est ce que l'homme fait de sa douleur, elle englobe ses attitudes, c'est-àdire sa résignation ou sa résistance à être emporté dans un flux douloureux, ses ressources physiques ou morales pour tenir devant l'épreuve. Elle n'est jamais le simple prolongement d'une altération organique, mais une activité de sens pour l'homme qui en souffre. Elle nomme l'élargissement de l'organe ou de la fonction altérés à toute son existence. Mais si la souffrance est inhérente à la douleur, elle est plus ou moins intense selon les circonstances. Un jeu de variations existe de l'une à l'autre. La souffrance est une fonction du sens que revêt la douleur, elle est en proportion de la somme de violence subie. Elle est une mesure intime de la douleur ressentie. Elle peut être infime ou tragique, elle n'est jamais mathématiquement liée à une lésion. 


\section{MODULATIONS SOCIALES ET CULTURELLES}

D'une condition sociale et culturelle à une autre, et selon leur histoire personnelle, les hommes ne réagissent pas de la même manière à une blessure ou à une affection identique. Leur expressivité n'est pas la même, ni leur seuil de sensibilité. Toutes les sociétés définissent implicitement une légitimité de la douleur qui accompagne des circonstances réputées physiquement pénibles. Une expérience cumulée du groupe amène à une attente de la souffrance coutumière imputable à l'événement. Une intervention chirurgicale ou dentaire, un accouchement, une affection, une blessure suscitent les commentaires avertis de ceux qui sont déjà passés par là ou connaissent «quelqu'un qui...». Un savoir profane double en permanence les expériences et associe chaque maladie, chaque lésion à une marge diffuse de souffrance dont l'expression individuelle se coule au sein de formes ritualisées. Quand une souffrance affichée paraît hors de proportion avec la cause et déborde le cadre traditionnel, on soupçonne la complaisance ou la duplicité. Là où il est socialement de rigueur d'endurer sa peine en silence, l'homme submergé qui donne libre cours à sa plainte encourt la réprobation. Cette entorse à la discrétion habituelle suscite des attitudes opposées à celles souhaitées par le malade: la compassion cède le pas à la gêne. À l'inverse, là où la ritualisation de la douleur appelle la dramatisation, on comprend mal celui qui intériorise sa peine et ne souffle mot à personne. La capacité du malade à affronter son épreuve sans montrer sa peine tranche avec les pleurs ou l'anxiété habituellement de rigueur. L'entourage est frustré dans son souci de prodiguer consolation et soutien. La plainte, en même temps qu'elle traduit la souffrance, a aussi valeur de langage qui confirme l'entourage dans le bien-fondé de sa présence, elle est une forme de communication. La douleur a ses rites d'expression que l'on ne transgresse pas sans le risque d'indisposer les bonnes volontés et de susciter le blâme ${ }^{3}$.

Les conditions d'existence modèlent les comportements jusqu'à un certain point mais il ne faut pas les transformer en stéréotypes venant occulter la singularité du malade. La douleur et ses manifestations ne relèvent pas seulement de la culture ou de la condition sociale. Ces formes d'influence n'existent qu'à travers les hommes qui les vivent et les significations qu'ils donnent à leur expérience. Aucun homme ne se réduit à une formule sociale ou culturelle, il est plutôt ce qu'il en fait. D'autres influences introduisent des ruptures et des continuités: cultures régionales et locales, rurales et urbaines, différences de générations, de sexe, etc. Chaque homme s'approprie les données de sa culture ambiante selon son histoire personnelle et les rejoue selon son style. La relation intime à la douleur ne met pas face à face une culture et une lésion, mais immerge dans une situation douloureuse particulière un homme dont l'histoire est unique même si la connaissance de son milieu, de son appartenance culturelle, donnent des indications précieuses sur le style de ses réactions.

En éprouvant sa douleur l'homme n'est pas le réceptacle passif d'un organe spécialisé obéissant à des modulations dont seule la physiologie pourrait rendre compte. La manière dont il intériorise sa culture, les valeurs qui sont les siennes, son rapport au monde, les circonstances particulières où il est plongé, composent un filtre spécifique. Face à la douleur, les différences rencontrées au sein d'une même culture sont parfois plus marquées que celles qui distinguent les cultures entre elles sous ce même rapport. La tâche des soignants consiste idéalement à répondre à la plainte sans présumer de son intensité, sans projeter leurs valeurs et leurs comportements propres pour juger de l'attitude de leurs patients. Cependant de nombreux travaux pointent à cet égard une fréquente sous-évaluation de la douleur. L'homme en bonne santé et actif est mal placé pour juger de la souffrance de l'autre, il risque la projection de sa psychologie propre au détriment du patient. Le stéréotype culturel empêche parfois d'entendre et de soulager la douleur. Or, ce ne sont pas seulement les malades qui intègrent leur douleur dans leur vision du monde, mais également les médecins ou les infirmières qui projettent leurs valeurs, et souvent leurs préjugés, sur ce que vivent les patients dont ils ont la charge (Zborowski, 1979). et la maladie à l'âge d'homme s'enracine aussi dans les inégalités de climat affectif ayant régi l'enfance. En comparaison, et de façon saisissante, les inégalités sociales de santé apparaissent de moindre importance. G. Menahem dégage ainsi plusieurs problèmes affectifs ayant pesé de longues années sur les individus. Le manque d'affection se traduit statistiquement par $49 \%$ de maladies supplémentaires, dont $57 \%$ touchant des affections de l'appareil respiratoire et digestif, et $76 \%$ pour des troubles d'expression psychique. Le conflit des parents correspond à $45 \%$ de déclarations de maladies supplémentaires. Leur absence prolongée aboutit à 36\%. Une maladie grave, un handicap ou un accident de la mère ou du père donnent une moyenne de $26 \%$ de déclarations supplémentaires dans le premier cas et de $23 \%$ dans le second. Les ouvriers ou les employés connaissent une sensibilité plus vive aux problèmes de l'enfance et aux difficultés ultérieures de santé. Statistiquement, ces personnes plus marquées que les autres par les conditions affectives de l'enfance sont aussi celles qui consultent le moins les médecins et sont les moins attentives à leur santé (Menahem, 1994; Menahem et Martin, 1994). Une étude américaine aboutit à des conclusions similaires. Sur 63 patients atteints de douleur chronique, $40 \%$ ont connu dans leur enfance une séparation de leurs parents, $23 \%$ ont été abandonnés ; $82 \%$ avouent un grave manque affectif dans leur enfance, $63 \%$ un rejet ouvert, et $19 \%$ une indifférence, un manque de disponibilité de leurs parents ; $33 \%$ ont été battus (Violin, 1990).

Au-delà de ces données, il convient de prendre en considération l'attitude des parents face à la souffrance. Une étude effectuée sur des adolescents affligés de

\section{SOUFFRIR COMME L'AUTRE NE SUFFIT PAS}

\section{À DISSIPER L'ÉLOIGNEMENT ET À NOUER D'EMBLÉE}

UNE COMMUNAUTÉ DE DESTIN,

\section{CAR LA DOULEUR ISOLE ET RETIENT CHACUN}

\section{DANS SES GRIFFES PARTICULIÈRES.}

Les conditions affectives de l'enfance échappent souvent à l'investigation de l'anthropologie mais elles sont essentielles pour comprendre le ressenti de la souffrance. Une étude de G. Menahem montre qu'un Français sur trois déclare avoir été confronté à des difficultés affectives durables lors de l'enfance. Devenues adultes ces personnes déclarent $43 \%$ de plus de maladies que celles qui n'en ont pas connu. Linégalité devant la souffrance douleur chronique bénigne a mis en relation leur attitude à celle de leur mère. Les mères ayant adopté une attitude de lutte en ne cessant d'encourager leurs enfants renforcent leur volonté de faire face. Ces adolescents, soutenus dans leur lutte active contre la douleur, disent la ressentir moins que leurs compagnons enclins à la passivité. Ces derniers vont rarement au terme des exercices prescrits par les médecins. Leur mère ont une attitude résignée et mêlent 
leur plainte à celle de leur enfant lors de ces activités. Leurs interactions soulignent surtout le caractère pénible et indépassable de la situation. La capacité de contenance de la mère, sa confiance envers les ressources de l'enfant, sont des données décisives pour comprendre la combativité de l'enfant et sa vulnérabilité ou non à la souffrance. Les comportements des parents fortifient ou affaiblissent l'enfant face à l'épreuve (Dunn-Geier et al., p. 23 et suiv.).

Un contexte d'instabilité affective, des parents détachés ou indisponibles se mobilisant soudain avec fougue à la moindre douleur de leur enfant associent en lui douleur et amour et alimentent une recherche répétitive de souffrance pour obtenir une reconnaissance de soi que les circonstances ordinaires peinent à établir. La famille est un lieu intense de socialisation où se construit le rapport au corps et au monde de l'enfant. Mechanic étudie l'incidence de l'attitude maternelle sur le rapport à la douleur de leurs enfants sur une population de 350 enfants de Madison dans le Wisconsin. Seul un pourcentage minime d'enfants vérifie l'hypothèse d'une étroite corrélation entre les comportements maternels et ceux de l'enfant. L'influence de la mère n'est pas un jeu de transparence reportant ses attitudes à la manière d'une décalcomanie. Plus l'enfant grandit et plus s'accroissent les interactions nourries avec les autres, étrangers à la sphère familiale. Les autorités éducatives se diversifient. En outre, la dynamique affective du groupe familial nuance l'ascendant respectif des uns et des autres sur l'enfant. Si les attitudes de la mère exercent un rôle prépondérant, celles du père, des grands-parents, des oncles ou des tantes, de la fratrie, ne sont pas sans interagir avec elles et sans bouleverser les intentions maternelles les plus ancrées. L'enfant n'est pas une page blanche où les autres écrivent ce qu'il doit être; il dispose d'une créativité propre qui le met en position d'acteur face aux modes d'impositions familiales. Il résiste aux modèles, il bricole avec ces influences pour se forger peu à peu ses manières propres de réagir à la maladie ou à la douleur. «Une mère surprotectrice, hypocondriaque, n'amène pas nécessairement son enfant à avoir des traits semblables (ou opposés)»(Mechanic, 1964, p. 453) ${ }^{4}$. Les ressources de l'enfant déjouent souvent la prégnance parentale.

La signification conférée par l'individu souffrant à l'épreuve endurée détermine également son rapport à la douleur. H. K. Beecher a observé une population de soldats blessés sur le front italien lors de la Seconde Guerre mondiale. Il est frappé par l'apaisement de ces hommes après leur évacuation hors du champ de bataille. Même gravement atteints, un tiers seulement des

LA TÂCHE DES SOIGNANTS CONSISTE IDÉALEMENT

À RÉPONDRE À LA PLAINTE SANS PRÉSUMER DE SON INTENSITÉ, SANS PROJETER LEURS VALEURS ET LEURS COMPORTEMENTS PROPRES

POUR JUGER DE L'ATTITUDE DE LEURS PATIENTS.

blessés demandent de la morphine pour soulager une douleur trop aiguë. Cette tolérance ne doit rien à l'état de choc car elle se prolonge par la suite. Beecher compare l'attitude de ces soldats à celle de patients civils ayant subi une intervention chirurgicale. Leurs lésions tissulaires sont moindres que celles affectant les soldats, mais leur demande d'analgésiques est nettement plus insistante et les plaintes sans commune mesure. La signification des douleurs n'est pas la même dans ces deux situations. Une blessure touchant un soldat habitué à se battre et à voir ses camarades blessés, ou périr, revêt une signification honorable: il a fait son devoir, pris sous les feux de l'ennemi, il n'a rien à se reprocher et surtout il est vivant. La blessure signifie un retrait des combats et une évacuation, avant le retour à la vie civile, avec une éventuelle pension. Le militaire blessé souffre moins d'un état de fait qu'il est accoutumé de longue date à considérer comme une conséquence possible de son activité que l'employé renversé par une voiture ou l'ouvrier dont le bras a été percuté par la machine qu'il manipulait. Loin du théâtre de la guerre, et rarement confronté à l'adversité, le civil est atteint au cœur de sa vie quotidienne, alors que rien ne l'a jamais préparé à affronter une telle situation (Beecher, 1956).

L'expérience clinique montre que selon la qualité de relation des soignants ou des proches qui se succèdent à son chevet, selon les moments du jour, les nouvelles qu'il reçoit sur son état, le sentiment qu'il a ou non d'être reconnu dans sa peine, le malade ne souffre pas de la même manière. La nuit accroît sa sensibilité et son angoisse, une présence attentive à son chevet la diminue. Une main sur un front, une parole d'apaisement sont souvent aussi nécessaires que les antalgiques, même si elles ne les remplacent pas. De même, les ressources personnelles d'imagination, de diversion, la détermination du caractère, contribuent à sa modulation.

\section{RÉCITS DE DOULEUR}

La maladie ou la douleur sont prises dans un «réseau sémantique» (Good, 1998; Good et al., 1992), une trame de mots, d'émotions, d'actions qui rend compte de la situation d'un patient à travers l'enchevêtrement de son discours avec celui des professionnels et d'autres patients, etc. Ce réseau de paroles mêle théories profanes et savantes, et génère comportements et actions à l'encontre de la pathologie. Son influence est diffuse, elle ne relève pas d'une injonction claire, mais d'une trame mouvante toujours en débat, toujours ouverte à de nouvelles contributions, pleine d'affectivité, d'éléments contradictoires, de récits édifiants, etc. Cette matrice de discours, plus univoque pour les médecins, nettement plus éclatée pour le patient, est un principe organisateur du rapport à la souffrance

La maladie est essentiellement dialogique. Elle est «synthétisée» [...] Elle est objectivée comme forme spécifique d'un trouble physiologique dans les présentations de cas et les conversations entre médecins. Mais l'objectivation peut rencontrer une résistance ou un désaccord des patients, des avocats ou des agents des compagnies d'assurances qui autorisent ou refusent les remboursements de certains traitements tout en négociant entre eux les constitutions de l' «objet» médical et la façon d'aborder le corps matériel. (Good, 1998, p. 352)

Ce discours demeure dans l'inachevé pour le patient toujours en quête de nouvelles informations ou de nouveaux traitements, prêt à recouper son histoire médicale à celle des autres, ou à remanier son analyse en fonction de la résonance en lui d'un témoignage qu'il vient d'entendre. Cette matrice est enracinée dans un contexte social, culturel, religieux, familial, voire de genre, mais elle est toujours ressaisie par l'histoire personnelle du patient et l'influence de ceux qui comptent pour lui. Le récit de douleur avec lequel le patient affronte son épreuve est un prisme qui module l'étendue de sa souffrance. Une voix singulière se mêle à une parole collective diffuse dans la tâche d'opérer un travail $\mathrm{du}$ sens pour humaniser la souffrance et en reprendre le contrôle. La signification de la maladie ou de la douleur s'établit sur une construction narrative du patient qui bricole avec les propos tenus par les médecins, les soignants, les autres malades, les témoignages glanés çà et là par ouï-dire, lectures ou reportages télévisés, ce que son histoire personnelle lui laisse pressentir 
de sa pathologie. Il y mêle son interprétation propre, ses remords, ses regrets, ses ressentiments, ses espoirs. La mise en récit de l'expérience est une tentative pour le patient d'accrocher du sens aux circonstances pour ne plus être emporté par elles et faire face à son épreuve selon une tonalité propre. Elle "prend ensemble», dit $\mathrm{P}$. Ricoeur, et «intègre en une histoire entière et complète les éléments multiples et dispersés» (Ricoeur, 1983, p.12). L'attribution d'un sens à sa douleur permet au patient de ne pas sombrer et de maintenir son regard sur les choses, de repousser la peur ou l'horreur. La trame de sens est une matière première de la culture qui fonctionne comme un bouclier protégeant de l'aspect brut et brutal du monde, elle retient l'homme dans un univers encore cohérent et prévisible (Le Breton, 1995, p. 107).

\section{CONSÉOUENCES DE LA DOULEUR}

Ce ne sont pas tant les circonstances qui induisent la souffrance que la manière dont elles sont interprétées et vécues. Le réel n'existe que par son appropriation par l'acteur. Le pire peut être tenu à distance tandis qu'une écorchure brise l'individu dans son envol. L'histoire de vie est déterminante pour comprendre l'incidence des événements ou d'une trame relationnelle sur l'individu.

Dans des circonstances maîtrisées par l'individu, la souffrance est insignifiante et permet alors de connaître des situations limites comme dans le sport extrême ou le body art par exemple. Elle devient même parfois un arrachement à soi-même où la souffrance est neutralisée mais où la violence des sensations éprouvées permet à certains, lors de suspensions par exemple, d'explorer les marges de la condition humaine, hors de tout contexte religieux, à vivre une intense expérience spirituelle. Une douleur choisie et maîtrisée par une discipline personnelle dans un but de révélation de soi ne contient qu'une parcelle dérisoire de souffrance, même si elle fait mal. Mais dans des circonstances différentes, par une sorte de sacrifice inconscient, elle offre le paradoxe de protéger l'individu d'une menace terrifiante de destruction de soi, la scarification délibérée est ainsi un paravent contre une souffrance intolérable. Il s'agit alors de se faire mal pour avoir moins mal. En revanche la souffrance déborde à l'infini la douleur dans le cas notamment de la torture, c'est-à-dire d'une douleur infligée par un autre sans être en mesure de l'en empêcher (Scarry, 1985; Vinar, 1989). Une douleur infligée de manière traumatique laisse une trace de souffrance même lorsqu'elle s'efface. Elle mutile une part du sentiment d'identité de l'individu qui n'arrive jamais tout à fait à oublier. Entre douleur et souffrance, les liens sont à la fois étroits et lâches selon les contextes, mais ils sont profondément significatifs et ouvrent la voie d'une anthropologie des limites. Si la douleur est un mot au singulier pour celui qui l'éprouve, elle revêt en fait une myriade de significations. S'il existe une pluralité de douleurs, c'est d'abord parce qu'il existe une pluralité de souffrances.

L'impact de la douleur connaît donc une virulence étroitement liée à la nature de la violation intime qu'elle contient. La douleur projette l'individu hors de lui-même, le révèle, pour le meilleur ou pour le pire, à des ressources propres dont il ignorait l'existence.

Si les liens entre douleur et souffrance sont multiples, ils sont toujours une équation du sens vécu par l'individu et du contexte dans lequel il est impliqué. Douleurs et souffrances sont toujours au pluriel. C'est la souffrance qui détruit l'homme. C'est pourquoi, s'agissant de malades ou d'accidentés, de victimes de traumatismes ou de tortures, la technique médicale est insuffisante, même si elle est fondamentale. La qualité de présence auprès du malade, l'accompagnement, l'instauration d'une confiance sans défaut en l'équipe soignante sont non moins essentiels. Le soulagement efficace de la douleur, parce qu'il implique simultanément une action sur la souffrance, sollicite une médecine centrée sur la personne et non seulement sur des paramètres biologiques. L'expérience des soins palliatifs montre combien l'accompagnement des malades en fin de vie a une valeur d'atténuation ou de suppression d'une douleur qui n'est jamais seulement "physique», mais touche l'homme en son entier, bouleverse son existence.

\section{Bibliographie}

BALINT, M. (1966). Le médecin, son malade et la maladie, Paris, Payot.

BEECHER, H.K. (1956). «Relationship of significance of wound to the pain experienced», Journal of American Medical Association, $\mathrm{n}^{\mathrm{o}} 161$.

DUNN-GEIER, B.J., et al. (1982). "Adolescent chronic pain: the ability to cope», Pain, $\mathrm{n}^{\circ} 26$, p. 23 et suiv.

GOOD, B. (1998). Comment faire de l'anthropologie médicale? Médecine, rationalité et vécu, Paris, Les Empêcheurs de penser en rond.

GOOD, M.-J., P. E. BRODWIN, B. GOOD et A. KLEINMAN (1992). Pain as human expérience: an anthropolological perspective, Berkeley, University of California Press.

LE BRETON, David (1995). Anthropologie de la douleur, Paris, Métailié.

LERICHE, René (1937). Chirurgie de la douleur, Paris, Masson.
MECHANIC, David (1964).«The influence of mothers on their children's health attitudes and behavior », Pediatrics, mars, p. 453.

MELSACK, R. et P. WALL (1989). Le défi de la douleur, Paris, Vigot.

MENAHEM, G. (1994). Problèmes de l'enfance, statut social et santé des adultes, Paris, Credes.

MENAHEM, G. et S. MARTIN (1994). "Quand l'enfance fait mal: liaisons entre événements de l'enfance et sensibilité des adultes aux maladies », Dialogues, n ${ }^{\circ} 142$.

RICOEUR, P. (1983). Temps et récit, Paris, Seuil.

SCARRY, E. (1985). The body in pain, Oxford, Oxford University Press.

ROY, R. et E. TUNKS (dir.) (1990). Chronic pain: psychosocial factors in rehabilitation, Baltimore, Williams and Wilkins.

THOMAS, W. et D.S. THOMAS (1970). The child in America, New York, Johnson Reprint Corp.

VIOLIN, A. (1990). «The process involved in becoming a chronic pain patient», dans R. ROY et E. TUNKS (dir.), Chronic Pain, Baltimore, Williams and Wilkins.

VINAR, M. et M. VINAR (1990). Exil et torture, Paris, Denoël

ZBOROWSKI, M. (1979). People in pain, San Francisco, Jossey-Bass.

\section{NOTES}

1. Mes recherches récentes m'ont amené à croiser ce thème sous différents angles. De manière plus générale dans $\mathrm{D}$. Le Breton, Anthropologie de la douleur (1995), Paris, Métailié. S'agissant du sport extrême ou des conduites à risque des jeunes générations dans Conduites à risque. Des jeux de morts au jeu de vivre (2002), Paris, Presses universitaires de France, collection «Quadrige»; et s'agissant des scarifications ou des attaques au corps chez les jeunes générations ou du body art dans La peau et la trace. Sur les blessures de soi (2003), Paris, Métailié.

2. Voir par exemple D. Le Breton (2002), Anthropologie du corps et modernité, Paris, Presses universitaires de France, collection «Quadrige» (1990), et La chair à vif. Usages médicaux et mondains du corps humain, Paris, Métailié, 1993.

3. Sur ce thème et pour un élargissement des propos tenus dans ce texte, je renvoie à D. Le Breton, Anthropologie de la douleur, op. cit.

4. Voir également M.-A. B. Veyra, «L'expression de la douleur de l'enfant lors d'interventions médicales: le rôle de la famille», dans J. Cook et A. Tursz, L'enfant et la douleur, Paris, Syros, 1998. 